\title{
Conversion from Deep Sedation to General Anesthesia in patients undergoing Endoscopic Retrograde Cholangiopancreatography: a series of cases
}

Eugênio Araújo Costa 1*, Priscila Ferreira de Lima e Souza 1, Mara Aparecida Maricato Poma 1, 2, Cláudia Regina Fernandes 1, Fred Olavo Aragão Andrade Carneiro 1, Miguel Ângelo Nobre e Souza 1, 3

${ }^{1}$ Hospital Universitário Walter Cantídio, Federal University of Ceará, Ceará, CE, Brazil.

2 Post-Graduation program in Pharmacology, Federal University of Ceará, Ceará, CE, Brazil.

${ }^{3}$ Post-Graduation program in Medical-Surgical Sciences, Federal University of Ceará, Ceará, CE, Brazil.

*Corresponding author: Eugênio Araújo Costa. pastor Samuel Munguba Street, 1290 - Rodolfo Teófilo. Zip Code: 60430-372 - Ceará, CE, Brazil. Phone: +55 (85) 9 9755-2244. E-mail: eugenioac3@hotmail.com.

Research Ethics Committee Approval (if necessary): Approved by the Universidade Federal do Ceará Ethics Committee (\#3.806.254).

Received on: Apr 30, 2021. Accepted on: May 10, 2021. Available online: May 14, 2021.

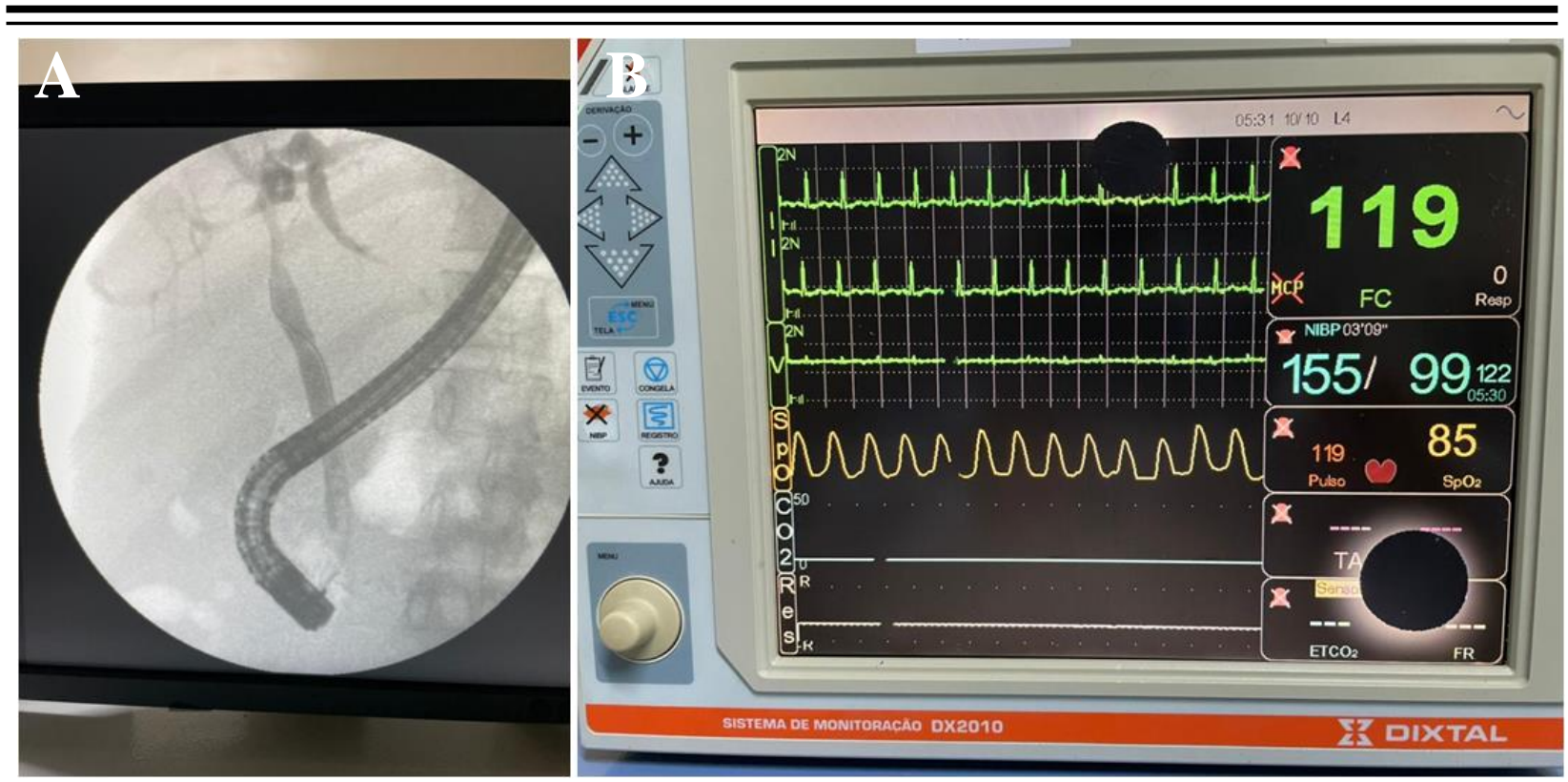

Figure 1. A. Radiographic record of ERCP showing an image of biliary stenosis due to liver transplantation. B. Dixtal multiparameter monitor showing hyposaturation in the therapeutic ERCP procedure in a patient under deep sedation.

In the present case series, these are the clinical aspects of two patients who underwent the endoscopic retrograde cholangiopancreatography (ERCP) surgical procedure with a focus on the treatment of biliary stenosis after liver transplantation. Patient 1 was 64 years old, $65 \mathrm{~kg}, 1.52 \mathrm{~m}$, body mass index (BMI) of 28.13, with a clinical picture of hypertension, Mallampatti score of 2, 
without predictors of difficult airway, submitted to liver transplantation by Cryptogenic Cirrhosis. Patient 2 was 47 years old, $62 \mathrm{~kg}, 1.51 \mathrm{~m}, \mathrm{BMI}$ of 27.19 , also hypertensive, non-insulindependent diabetic, with Mallampatti score 2, without predictors of difficult airway, submitted to liver transplantation due to cirrhosis by virus of the Hepatitis C.

Both patients underwent the ERCP procedure, under the protocol of deep sedation. It consisting of complete monitoring (Oximetry (SPO2) by continuous plethysmography, verification of non-invasive blood pressure every 5 minutes and continuous cardioscopy), under oxygen supply by nasal catheter, followed by intravenous administration of Fentanyl $2 \mu / \mathrm{kg}+$ Ketamine $5 \mathrm{mg}+$ Midazolam $5 \mathrm{mg}$, for the purpose of anesthetic induction and Propofol (75-200 $\mu \mathrm{g} / \mathrm{kg} /$ min) in a fresenius kabi infusion pump (by software programmed for target TCI - Target Controlled Infusion) in the range of $1.2-2.2 \mu \mathrm{g} / \mathrm{ml}$ ) for the purpose of induction and anesthetic maintenance.

During the surgical procedure, a patient 1 presents episodes of desaturation (SPO2 <90\%), making it necessary to frequently perform manual clearing maneuvers of the airways (hyperextension of the head and chin elevation), interrupting the ERCP frequently and impairing the completion of the exam. Patient 2 presented with an episode of sustained hyposaturation (SPO2 <90\% greater than 30 seconds), not reversed with airway maneuvers. For both cases of conversion to general anesthesia, it was essential to ensure patient safety and clinical control of the ERCP surgical procedure.

The general anesthesia induction protocol for both patients was followed by intravenous administration of

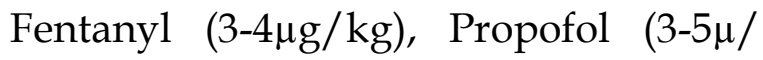
$\mathrm{kg})$ and Cisatracurium $(0.1 \mathrm{mg} / \mathrm{kg})$. For anesthetic maintenance, Sevoflurane (inspired fraction of 1.5-2\%) and Atropine $(20 \mu \mathrm{g} / \mathrm{kg})$ and Neostigmine $(40 \mu \mathrm{g} / \mathrm{kg})$ were administered to reverse neuromuscular block at the end of the procedure.

Through a general anesthesia protocol, it was found that ERCP surgical procedures were successfully performed, without further interruptions or clinical complications, such as other episodes of hypoxemia. In view of the clinical characteristics of the patients, it is important to emphasize that an ERCP is an essential surgical procedure in the diagnosis and treatment of a variety of pancreaticbiliary disorders, for example, for the assessment of cholelithiasis cases until neoplasms [1], as well as repair biliary stenosis.

The complications related to ERCP are known to be pancreatitis, hemorrhage, cholangitis, infection. However, $4-16 \%$ of complications are associated with cardiopulmonary events 
such as hypoxemia, bronchoaspiration, hypotension and arrhythmias, especially when evaluating the type of anesthetic approach used, whether deep sedation or general anesthesia [2].

It is known that patients with risk factors for sedation have a conversion rate to general anesthesia of $10 \%$. Among the previous clinical factors that stand out for the choice of general anesthesia as a clinical conduct for ERCP clinical patients, sleep apnea, the stop-bang score (sleep apnea assessment) $>3$, presence of ascites, patients stand out with BMI> 35, Mallampati score 4, Alcoholics (> 4 drinks / day in men and $>3$ drinks / day in women), history of chronic obstructive pulmonary disease and ASA classification> 3 [3].

In this cases series, as patients not fulfilled the above risk factors for complications during deep sedation. However, both patients evolved with respiratory complications, impairing the safety of patients under sedation, which led to the conversion to general anesthesia. We need to critical thinking about the current risk factors for complications in sedation are insufficient to define the choice of the best anesthetic technique that is individualized to the profile of the patient clinician.

\section{References}

[1] Hormati A, Aminnejad R, Saeidi M, Ghadir MR, Mohammadbeigi A, Shafiee $\mathrm{H}$. Prevalence of Anesthetic and
Gastrointestinal Complications of Endoscopic Retrograde Cholangio pancreatography. Anesth Pain Med. 2019 Aug 26;9(4):e95796. doi: 10.5812/aapm.95796. PMID: 31754612; PMCID: PMC6825367.

[2] Johnson KD, Perisetti A, Tharian B, Thandassery R, Jamidar P, Goyal H, Inamdar S. Endoscopic Retrograde Cholangiopancreatography - Related Complications and Their Management Strategies: A "Scoping" Literature Review. Dig Dis Sci. 2020 Feb;65(2):361375.

[3] Smith ZL, Mullady DK, Lang GD, Das KK, Hovis RM, Patel RS, Hollander TG, Elsner J, Ifune C, Kushnir VM. A randomized controlled trial evaluating general endotracheal anesthesia versus monitored anesthesia care and the incidence of sedation-related adverse events during ERCP in highrisk patients. Gastrointest Endosc. 2019 Apr;89(4):855-862.

Conflict of interest: The author declares no conflicts of interest.

Acknowledgements: None.

Funding: None.

How to cite this article: Costa EA, Lima e Souza PFL, Poma MAM, Fernandes CR, Carneiro FOAA, Nobre e Sousa MA. Conversion from Deep Sedation to General Anesthesia in patients undergoing to Endoscopic Retrograde Cholangio pancreatography: a series of cases. Brazilian Journal of Case Reports. 2021 Abr-Jun;01(2):42-44. 